\title{
ANÁLISES VISUAL E VOLUMÉTRICA POR RESSONÂNCIA MAGNÉTICA DAS FORMAÇÕES HIPOCAMPAIS EM UM GRUPO DE PACIENTES COM DIAGNÓSTICO CLÍNICO DE EPILEPSIA DO LOBO TEMPORAL
}

\author{
ENNIO ROGACHESKI*, SÉRGIO MAZER*, DIRCEU RODRIGUES**, \\ PAULO ROGERIO MUDROWICH DE BITTENCOURT ***
}

\begin{abstract}
RESUMO - Visando comparar a sensibilidade da avaliação volumétrica com a análise visual na avaliação por ressonância magnética $(\mathrm{RM})$ das formações hipocampais de pacientes com epilepsia do lobo temporal refratária e candidatos à lobectomia temporal, estudamos 153 casos com diagnóstico clínico de epilepsia do lobo temporal, utilizando um equipamento de 0,5 Tesla, com técnica de inversion-recovery ponderada em T1, com cortes de 5 $\mathrm{mm}$ no plano coronal. Houve boa concordância entre a análise visual prospectiva e outra retrospectiva, realizada por dois observadores independentes $(C=0,748$ e 0,720$)$. Houve também concordância entre a análise retrospectiva dos dois observadores $(C=0,733)$. Houve ainda concordância genuína $(C=0,788)$ entre os resultados das análises quantitativa e qualitativa realizadas prospectivamente. A análise quantitativa demonstrou uma tendência nãosignificativa a lateralizar mais casos de atrofia hipocampal presumida clinicamente. Nossos resultados confirmam a confiabilidade da análise visual qualitativa e indicam a utilidade da volumetria hipocampal como uma medida suplementar, objetiva e quantitativa, de esclerose hipocampal.
\end{abstract}

PALAVRAS-CHAVE: epilepsia do lobo temporal, ressonância magnética, hipocampo.

\section{Visual and volumetric analyses of the hippocampal formations of a group of patients with clinical diagnosis of temporal lobe epilepsy}

\begin{abstract}
The purpose of this study is to test the sensitivity of the volumetric analysis compared to the visual analysis of the hippocampal formations of a group of 153 patients with clinical diagnosis of temporal lobe epilepsy and candidates to temporal lobectomy, evaluated by magnetic resonance (MR), using a 0.5 Tesla machine and inversion-recovery $\mathrm{T} 1$-weighted images and $5 \mathrm{~mm}$ coronal slices. There was agreement between the prospective visual analysis and another retrospective visual analysis carried out by two independent observers $(\mathrm{C}=0.748$ and $\mathrm{C}=0.720$ ). There was also agreement between the retrospective analysis of the two investigators $(\mathrm{C}=0.733)$. There was genuine agreement $(\mathrm{C}=0.788)$ between the results of the qualitative and quantitative analyses carried out prospectively. Volume measurements showed a nonsignificant tendency to lateralize more cases of clinically presumed hippocampal atrophy. Our results confirm the reliability of a qualitative visual analysis and indicate the utility of hippocampal volumetry as a supplementar, objective and quantitative measure of hippocampal sclerosis.
\end{abstract}

KEY WORDS: temporal lobe epilepsy, magnetic resonance imaging, hippocampus.

*Neuro-radiologista da Liga das Senhoras Católicas de Curitiba e do Hospital de Clínicas da Universidade Federal do Paraná (UFPR); **Professor Senior da Disciplina de Radiologia da UFPR (Orientador); ***Coordenador do Grupo de Cirurgia de Epilepsia do Hospital N. Senhora das Graças de Curitiba (Co-orientador). Este trabalho representa uma parte da dissertação submetida pelo primeiro autor ao Mestrado de Medicina Interna (Área: Radiologia) do Departamento de Clínica Médica da UFPR, em 1995 e foi realizado no Departamento de Ressonância Magnética da Liga das Senhoras Católicas de Curitiba. Aceite: 28-abril-1998.

Dr. Ênio Rogacheski - Rua Prof. Arnaldo Alves de Araújo 138 - 80.740-430 Curitiba PR - Brasil. 
Epilepsia do lobo temporal (ELT) é o diagnóstico mais comum entre os portadores de epilepsia que têm crises resistentes ao tratamento clínico e que correspondem a 10-30 \% do total de casos ${ }^{1}$. A crise parcial complexa é tipicamente a mais refratária². Um subgrupo de pacientes tem a síndrome da ELT mesial, com crises originadas na formação hipocampal, amígdala e tecidos vizinhos. $\mathrm{O}$ achado mais frequente de ELT mesial é a chamada esclerose mesial temporal (EMT), também chamada esclerose hipocampal (EH) ${ }^{3-6}$ ou, menos comumente, esclerose do corno de Ammon (ECA). Histologicamente, a EMT caracteriza-se por graus variáveis de perda ou depleção neuronal e substituição gliótica, ou seja: trata-se de lesão esclerótica, cicatricial ou atrófica acometendo as porções mesiais do lobo temporal, principalmente, o hipocampo, mas também outras estruturas, como a amígdala e o unco, conforme estabelecido por Falconer e Taylor, em 1968 ${ }^{7}$. Esses autores preferem o termo esclerose mesial temporal aos demais (esclerose hipocampal e esclerose do corno de Ammon), pelo fato de poder existir esclerose fora do hipocampo, como na amígdala, e por ser puramente descritivo, sem implicações etiológicas ou funcionais. Macroscopicamente, os lobos temporais podem ser divididos em neocórtex temporal e estruturas mesiais, que incluem o unco, a amígdala, o hipocampo e o giro para-hipocampal ${ }^{8,9}$.

A anatomia das estruturas têmporo-mesiais é melhor demonstrada no plano coronal, com cortes coronais ou coronais-oblíquos, tanto nas peças anatômicas, quanto nos cortes de ressonância magnética (RM). O hipocampo é estrutura complexa do lobo temporal mesial e faz parte do sistema límbico e do circuito da memória ${ }^{10-13}$. Por ser estrutura visível pela RM (Fig 2), sua avaliação pode ser importante, não só em epilepsia, mas também na doença de Alzheimer, esquizofrenia e outras doenças amnésticas ${ }^{14-16}$. O termo formação hipocampal $(\mathrm{FH})$ refere-se a uma estrutura ou a uma unidade funcional complexa, encontrada no lobo temporal mesial, constituída pelo hipocampo, ou corno de Ammon, subículo, giro denteado e córtex entorrinal ${ }^{12}$. Frequentemente, na literatura, emprega-se o termo formação hipocampal, em vez de hipocampo ${ }^{11,17,18}$, o que é também adotado neste estudo. Macroscopicamente, o hipocampo, que mede cerca de 4,5 cm no seu diâmetro longitudinal, possui três partes: cabeça, também denominada pé do hipocampo, corpo e cauda ${ }^{9}$. Detalhes da anatomia destas estruturas podem ser encontrados na literatura ${ }^{1,2,9,13,19,20}$.

Anteriormente, a cabeça do hipocampo separa-se da amígdala pelo recesso uncal do corno temporal do ventrículo lateral. Deve-se lembrar, ainda, que o hipocampo representa estrutura de córtex invertida, na qual se observa substância branca (alveus) envolvendo estruturas de substância cinzenta. Outras vias de substância branca relacionadas às estruturas têmporo-mesiais são a fímbria e o fórnix, assim como suas continuações vestigiais ao redor do corpo caloso. A sinonímia do sistema límbico é variável, o que dificulta o seu estudo ${ }^{11,13}$. Microscopicamente, o hipocampo ou corno de Ammon possui quatro zonas distintas (CA1, CA2, CA3 e CA4), a partir do subículo , em direção ao giro denteado, segundo as diferenças histológicas de sua principal camada celular. Considerando a sensibilidade à hipóxia, o setor CA1, também chamado setor de Sommer, é considerado setor vulnerável; CA2 e CA3, em conjunto, chamados setor de Spielmeyer, ou setor resistente e o CA4, adjacente ao giro denteado, é o setor de Bratz ou setor de vulnerabilidade média ${ }^{13,21}$. No hipocampo propriamente dito, ou corno de Ammon, as camadas celulares mais comumente envolvidas no processo esclerótico da EMT são o setor CA1 e, em menor grau, o CA4.

Esclerose mesial temporal consiste em perda neuronal, demonstrada nos métodos de neuroimagem, sobretudo pela RM, pela atrofia e alteração da morfologia interna, associadas a proliferação gliótica, que se traduz por por hipointensidade em T1 e hiperintensidade em T2, nas imagens de $\mathrm{RM}^{3,4}$.

A etiologia da EMT é tema de controvérsia na literatura ${ }^{22}$. Esclerose hipocampal pode ter várias causas e tem sido fortemente associada a antecedentes de convulsões febris prolongadas na infância ${ }^{9,22}$. As convulsões repetidas ao longo da vida não parecem causar aumento mensurável da atrofia da amígdala e da formação hipocampal. Especula-se, pois, que a EMT seja causa da epilepsia e não consequência dela ${ }^{23}$. Sabe-se que há boa correlação entre os achados de RM, com cortes finos 
Quadro 1. Aspectos da esclerose hipocampal.

\begin{tabular}{ll}
\hline RM da esclerose hipocampal & Correlação histológica \\
\hline Atrofia unilateral & Atrofia hipocampal \\
Perda da morfologia interna & $\begin{array}{l}\text { Perda de neurônios em CA1, CA3, e CA4 e } \\
\text { substituição gliótica }\end{array}$ \\
Aumento do sinal em T2 & Gliose \\
Redução do sinal em T1 & Gliose \\
\hline
\end{tabular}

Reproduzido e adaptado de Jackson et al. ${ }^{4}$

e de alta resolução, e critérios sugestivos de esclerose hipocampal. O binômio atrofia hipocampal aumento de sinal em T2, no interior da formação hipocampal, constituiu, durante muito tempo, a chave para o diagnóstico de EMT, pela análise visual. Recentemente, descreveu-se também a redução de sinal, como indicativo de gliose, em cortes finos $(5 \mathrm{~mm})$ das formações hipocampais, com técnica inversion-recovery ${ }^{4}$. Sinal alterado (em T1 e/ou T2) e a morfologia hipocampal alterada (atrofia e/ ou perda da estrutura interna) constituem os aspectos diagnósticos de esclerose hipocampal, pela análise visual em cortes de RM (Quadro 1) ${ }^{4}$. Enfatiza-se também um grau variável de achatamento do hipocampo como sinal indicativo de alteração morfológica associado à atrofia hipocampal ${ }^{9}$. Considerando-se os métodos de neuro-imagem estrutural, a RM é mais sensível do que a tomografia computadorizada (TC) para a avaliação das estruturas têmporo-mesiais, constituindo-se no método de escolha em portadores de epilepsias refratárias ao tratamento clínico, pois, além de permitir a detecção de causas lesionais ou evidências de tecido anômalo, demonstra os achados característicos da EMT, em candidatos a lobectomia temporal ${ }^{3}$. A análise visual, executada em cortes realizados segundo princípios específicos, demonstra assimetrias e é consagrada na literatura especializada ${ }^{1-4,9}$. Alguns grupos enfatizam que a análise objetiva, volumétrica, das formações hipocampais seria uma medida mais sensível que a análise subjetiva e qualitativa, para a lateralização em candidatos cirúrgicos avaliados por ELT intratável $\mathbf{6}^{6,14,18,22,24}$.

O objetivo do presente estudo é avaliar a presença de atrofia das formações hipocampais, como elemento de lateralização da área epileptogênica, em um grupo de pacientes com o diagnóstico clínico presumido de ELT, comparando os resultados de uma análise visual, com os de uma análise volumétrica das formações hipocampais, obtidas pela RM. A proposta central é testar a confiabilidade da análise volumétrica, utilizando equipamento de 0,5 Tesla, com técnica de inversion-recovery, ponderada em $\mathrm{T}^{9}$, com cortes de $5 \mathrm{~mm}$ de espessura, no plano coronal (Fig 1). A hipótese central do estudo é que a volumetria das formações hipocampais, com cortes de $5 \mathrm{~mm}$ de espessura, em equipamento de RM de médio campo magnético (0,5 Tesla), apresenta eficácia semelhante a um método consagrado, que é a análise visual. A oportunidade e a relevância do estudo, sobretudo quando considerado dentro de contexto mais amplo, como o de um programa de cirurgia de epilepsia, parecem evidentes, devido à necessidade de seleção de candidatos ao tratamento cirúrgico, com vistas à cura ou melhor controle de quadros epilépticos refratários.

\section{MATERIAL E MÉTODO}

O presente estudo, do tipo observacional, prospectivo e não-controlado, foi desenhado com o intuito de se avaliar o rendimento da hipocampometria, comparado ao da análise visual de formações hipocampais. Foram selecionados 153 casos do universo de pacientes com diagnóstico clínico presumido de ELT de difícil controle medicamentoso, encaminhados ao Departamento de Ressonância Magnética da Liga das Senhoras Católicas de Curitiba, estado do Paraná, Brasil, entre maio de 1992 e junho de 1995. O critério básico de inclusão foi, por conseguinte, o diagnóstico clínico de ELT de difícil controle medicamentoso. Os critérios de exclusão da amostra incluem: presença de lesão ocupando espaço ou tecidos anômalos; artefatos grosseiros nas imagens de RM; assimetrias dos cortes por posicionamento inadequado; e aqueles pacientes cujo diagnóstico clínico sugeria ELT, 


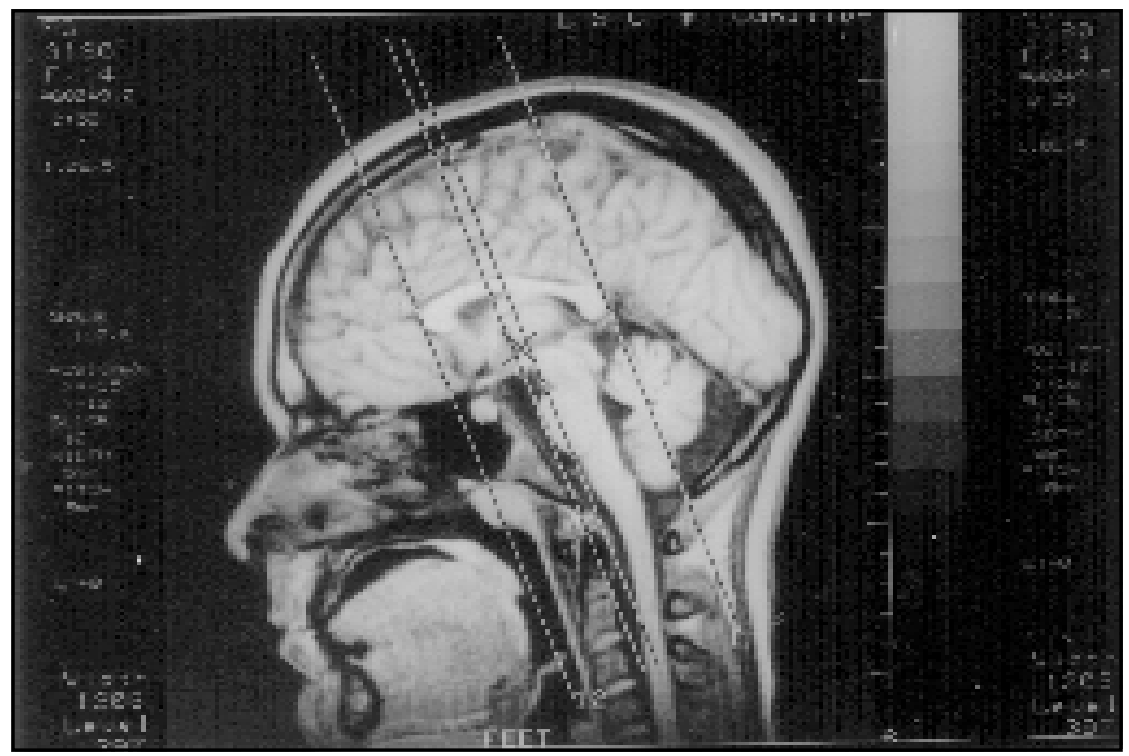

Fig 1. Corte sagital mediano de RM, com técnica FE ponderada em T1, indicando o planejamento dos cortes coronais, paralelos ao tronco encefálico ${ }^{4}$, destinados às análises visual e volumétrica dos casos do estudo.

sendo posteriormente classificados como portadores de epilepsia extratemporal (focal ou generalizada). Os casos selecionados para esta amostra, além do número do exame de RM do Serviço, receberam número sequencial para o presente estudo ( $\mathrm{n}=153$ ). A nossa casuística era constituída de 77 casos do sexo masculino e 76 do sexo feminino, com idade variando entre 3 e 70 anos (média 28,95 12,34 e mediana 30 anos). Considerando a faixa etária, a distribuição dos casos foi a seguinte: 20 casos (13\%) entre 0 e 14 anos; 56 casos $(36,6 \%)$ entre 15 e 29 anos; 68 casos $(44,4 \%)$ entre 30 e 49 anos e apenas 9 casos $(5,8 \%)$ com 50 anos ou mais.

O Protocolo ELT desenhado para o estudo por RM deste grupo de pacientes incluía: sequência field-echo ponderada em T1, no plano sagital, com TR de $300 \mathrm{~ms}$, TE de $20 \mathrm{~ms}$, com 11 cortes de $5 \mathrm{~mm}$ de espessura, para o planejamento das demais sequências, seguida de sequência de cortes axiais spin-echo ponderados em T2 e densidade de prótons, com TR de $2.500 \mathrm{~ms}$, TE de $20 \mathrm{~ms}$ e $90 \mathrm{~ms}$, com 12 cortes de $8 \mathrm{~mm}$ de espessura, para o screening do encéfalo, visando à detecção de lesões fora das regiões têmporo-mesiais. A seguir, era obtida uma sequência inversion-recovery, ponderada em T1, no plano axial, com inclinação para os lobos temporais ${ }^{4}$, com TR de $2.000 \mathrm{~ms}$, TE de $20 \mathrm{~ms}$ e TI de $600 \mathrm{~ms}$, com 12 cortes de $5 \mathrm{~mm}$ de espessura. Por fim, era obtida sequência inversion-recovery, ponderada em $\mathrm{T} 1$, no plano coronal, obtido ao longo do eixo do tronco encefálico ${ }^{4}$, conforme demonstrado na Fig 1, com TR de $2.500 \mathrm{~ms}$, TE de $20 \mathrm{~ms}$ e TI de $600 \mathrm{~ms}, 12$ cortes de $5 \mathrm{~mm}$ de espessura e pitch de $5 \mathrm{~mm}$, seguida de outra sequência spin-echo, ponderada em T2, com TR de $2.500 \mathrm{~ms}$, TE de $110 \mathrm{~ms}$ e 12 cortes de $5 \mathrm{~mm}$ de espessura e $5 \mathrm{~mm}$ de pitch. Estas duas sequências coronais eram programadas de maneira a se obter a máxima congruência possível, para a análise visual comparativa das alterações morfológicas e dos sinais, assim como das assimetrias.

Todos os exames de RM foram realizados em equipamento de 0,5 Tesla, com magneto supercondutivo, da marca Shimadzu (SMT - 50X). Todas as imagens obtidas foram documentadas em filmes radiográficos. Utilizando-se apenas a sequência de cortes coronais inversion-recovery, foram realizadas análises visual e volumétrica prospectivas e comparadas entre si, para se avaliar o grau de concordância entre ambas. Foi comparada também a análise visual prospectiva com outra retrospectiva, realizada por dois observadores independentes e com experiência semelhante, sendo o primeiro autor deste estudo denominado observador 1 e o primeiro coautor, observador 2. De acordo com a análise visual, considerando-se exclusivamente os aspectos de atrofia hipocampal ${ }^{3,4,9}$, foram determinados os seguintes subgrupos: classe $\mathrm{D}$ - atrofia à direita; classe $\mathrm{E}$ - atrofia à esquerda; e classe I - indeterminada, cuja análise visual era considerada não-lateralizante (Fig 2). A análise volumétrica era realizada nos mesmos cortes coronais inversion-recovery ponderados em T1, diretamente no 


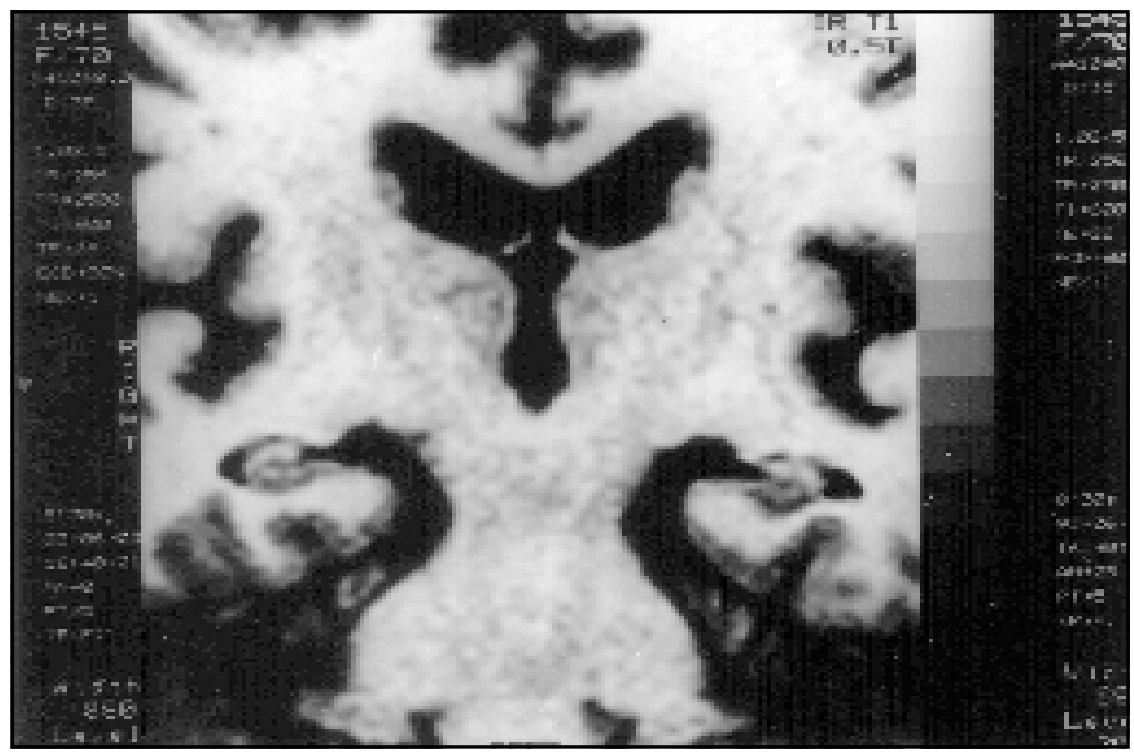

Fig 2. Corte de RM no plano coronal, com técnica IR em T1, ao nível do corpo dos hipocampos (Caso 12 - classe I). Note-se a morfologia característica dos hipocampos, visualmente simétricos.

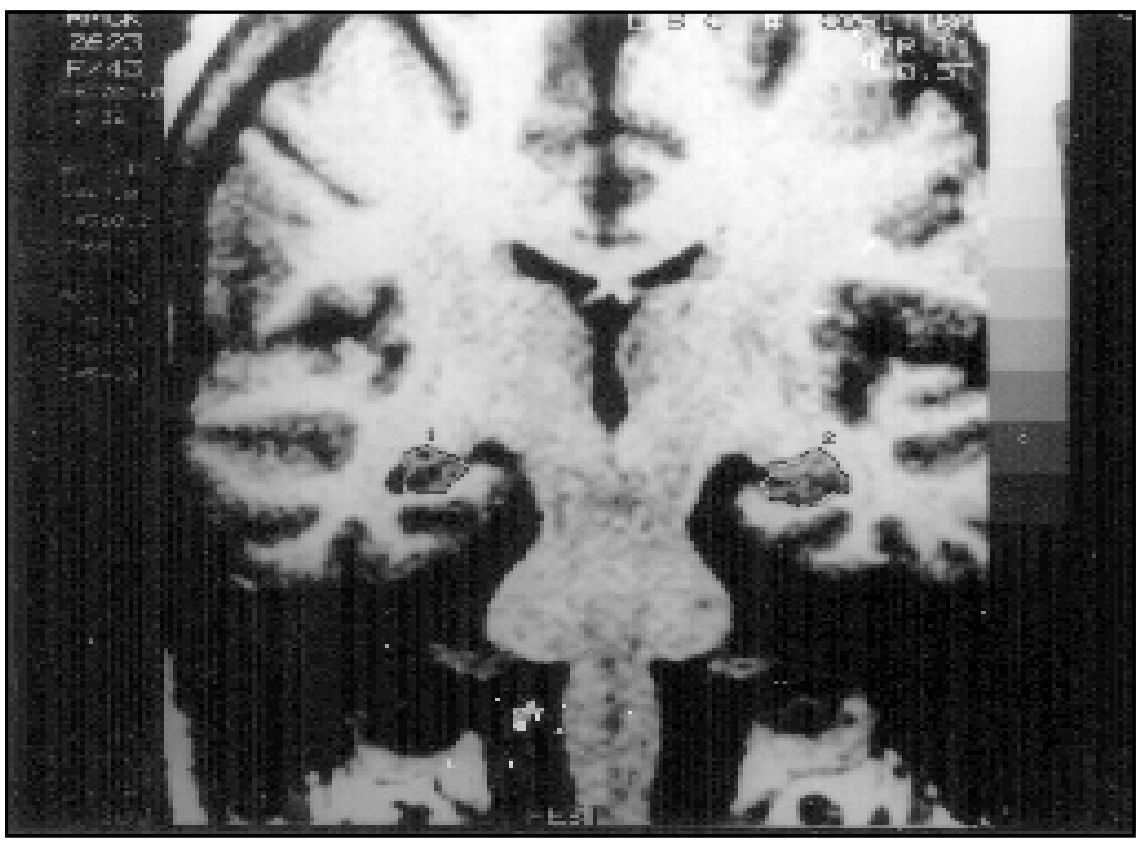

Fig 3. Corte coronal de RM, com técnica IR ponderada em T1, ilustrando a delimitação da área correspondente ao corte, ao nível do corpo dos hipocampos, para o estudo volumétrico (Caso 29 - classe D). Note-se a assimetria das formações hipocampais, qualitativa e quantitativamente menor à direita, além da perda da morfologia. 
monitor do equipamento, traçando-se manualmente um ROI (region of interest), para cada corte ${ }^{25}$, conforme ilustra a Fig 3. Somando-se os valores das áreas de cada corte, obtinha-se uma área total, para cada lado (em $\mathrm{mm}^{2}$ ), sendo multiplicado por 0,005 , para se obter o volume em $\mathrm{cm}^{3}$. A seguir, subtraía-se o valor do volume do lado esquerdo daquele obtido para o lado direito (D-E) e obtinha-se um valor diferencial. Considerando-se critérios estabelecidos pela literatura ${ }^{25}$, foram utilizados os seguintes pontos de corte: $-0,2 \mathrm{~cm}^{3}$ e $0,5 \mathrm{~cm}^{3}$.

Pelo fato de não dispormos de um grupo-controle, não aplicamos desvio-padrão a esses valores, para a lateralização da atrofia hipocampal. Foi considerada, portanto, a seguinte faixa de indeterminação da atrofia hipocampal (classe I): valores entre $-0,2 \mathrm{~cm}^{3}$ e $0,5 \mathrm{~cm}^{3}$. Valores iguais ou menores que $-0,2 \mathrm{~cm}^{3}$ indicavam atrofia da FH direita (classe D) e valores iguais ou maiores que $0,5 \mathrm{~cm}^{3}$, atrofia esquerda (classe E).

A análise estatística dos dados foi feita em duas etapas: uma análise descritiva dos dados e uma análise comparativa. A análise descritiva, além de informações sobre dados epidemiológicos da amostra, apresenta os resultados das análises visual e volumétrica prospectivas, assim como da análise visual retrospectiva dos dois observadores. A análise comparativa dos dados obtidos objetivou verificar as relações existentes entre as diversas observações realizadas. Calculou-se o valor da estatística qui-quadrado, para a determinação do grau de significância destas relações e do coeficiente de contingência, que fornece o valor das correlações entre as observações. Determinou-se ainda o percentual de observações concordantes entre as diversas análises. Para efeito de determinação de significâncias, utilizou-se como referência o nível de significância de $5 \%$.

\section{RESULTADOS}

A análise visual prospectiva apresentou 40 casos $(26,1 \%)$ na classe D, 37 casos $(24,2 \%)$ na classe $\mathrm{E}$ e $76(49,7 \%)$, na classe I. Nota-se que o número de casos na classe I é quase equivalente à soma dos casos das classes D e E. A análise volumétrica prospectiva também mostrou resultados semelhantes aos da análise visual: 41 casos $(26,8 \%)$ na classe D, 40 casos $(26,1 \%)$ na classe E e 72 casos $(47,1 \%)$ na classe I. A análise visual retrospectiva realizada pelo observador 1 mostrou 36 casos $(23,5 \%)$ na classe D, 32 casos $(20,9 \%)$ na classe E e $85(55,6 \%)$ na classe I. A análise visual retrospectiva do observador 2 demonstrou 34 casos (22,2\%) na classe D, 29 casos (19\%) na classe E e 90 casos $(58,8 \%)$ na classe I. Os resultados da análise comparativa das análises visual e volumétrica prospectivas são demonstrados na Tabela 1 . O coeficiente de contingência é 78,8\%, e o nível de significância é menor que $0,01 \%$. Isto significa que existe uma relação genuína entre as duas análises e a associação não é resultado do acaso. Existe uma concordância de 93,46\% entre as observações. A relação entre a análise visual retrospectiva dos dois observadores e a análise visual prospectiva consta da Tabela 2. O coeficiente de contingência para o observador 1 é $74,8 \%$, e o nível de significância é menor que $0,01 \%$. Isto implica na existência de uma relação genuína entre as duas análises. A associação não é, portanto, resultado do acaso. Existe concordância de 86,3\% entre as observações. Para o observador 2, o coeficiente de contingência é 72\%, e o nível de significância é menor que $0,01 \%$. Pode-se inferir uma relação genuína entre ambas as análises. A associação não é, portanto, resultado do acaso e existe concordância de $82,4 \%$ entre as observações. A relação entre

Tabela 1. Relação entre as análises visual e volumétrica, das formações hipocampais, realizadas prospectivamente.

\begin{tabular}{ccccc}
\hline \multicolumn{5}{c}{ Análise volumétrica } \\
\hline Análise visual & D & I & E & Total \\
\hline D & 39 & 1 & 0 & 40,00 \\
I & 2 & 69 & 5 & 76,00 \\
E & 0 & 2 & 35 & 37,00 \\
Total & 41 & 72 & 40 & 153,00 \\
\hline
\end{tabular}

Qui-quadrado=250,39; $\mathrm{p}<0,0001 ; \mathrm{C}=0,788 ;$ Conc. $=93,46 \%$.

$\mathrm{D}$, atrofia à direita; E, atrofia à esquerda; I, indeterminado. 
Tabela 2. Relação entre a análise visual prospectiva e a análise visual retrospectiva dos 2 observadores.

\begin{tabular}{|c|c|c|c|c|c|c|c|}
\hline \multirow[b]{2}{*}{ Análise visual } & \multicolumn{3}{|c|}{ Observador $1^{*}$} & \multicolumn{3}{|c|}{ Observador $2 * *$} & \multirow[b]{2}{*}{ Total } \\
\hline & $\mathrm{D}$ & I & $\mathrm{E}$ & $\mathrm{D}$ & I & $\mathrm{E}$ & \\
\hline $\mathrm{D}$ & 33 & 7 & 0 & 30 & 10 & 0 & 40,00 \\
\hline I & 3 & 70 & 3 & 3 & 70 & 3 & 76,00 \\
\hline E & 0 & 8 & 29 & 1 & 10 & 26 & 37,00 \\
\hline Total & 36 & 85 & 32 & 34 & 90 & 29 & 153,00 \\
\hline
\end{tabular}

*Qui-quadrado=193,82; $<<0,0001 ; \mathrm{C}=0,748 ;$ Conc. $=86,30 \%$.

**Qui-quadrado $=164,37 ; \mathrm{p}<0,0001 ; \mathrm{C}=0,720 ;$ Conc. $=82,40 \%$.

$\mathrm{D}$, atrofia à direita; E, atrofia à esquerda; I, indeterminado.

Tabela 3. Relação entre as análises visuais retrospectivas dos 2 observadores.

\begin{tabular}{ccccc}
\hline \multicolumn{5}{c}{ Observador 2 } \\
\hline Observador 1 & D & I & E & Total \\
\hline D & 29 & 7 & 0 & 36,00 \\
I & 5 & 76 & 4 & 85,00 \\
E & 0 & 7 & 25 & 32,00 \\
Total & 34 & 90 & 29 & 153,00 \\
\hline
\end{tabular}

Qui-quadrado $=177,92 ; \mathrm{p}<0,0001 ; \mathrm{C}=0,733$; Conc. $=84,97 \%$

$\mathrm{D}$, atrofia à direita; E, atrofia à esquerda; I, indeterminado.

os resultados da análise visual retrospectiva dos dois observadores consta da Tabela 3. Estes resultados permitem verificar que o observador 1 tende a lateralizar mais que o observador 2 , mas não existe uma diferença estatística entre as percentagens de concordância das análises dos dois observadores. $\mathrm{O}$ nível de significância obtido nesta comparação foi $\mathrm{p}=0,4319$. Este resultado pode ser confirmado pela análise de correlação demonstrada pela Tabela 3. O coeficiente de contingência é $73,3 \%$, e o nível de significância é menor que $0,01 \%$. Isto demonstra que existe uma relação genuína entre as duas análises. A associação não é, portanto, resultado do acaso e existe uma concordância de $84,97 \%$ das observações. A relação entre a análise visual retrospectiva dos dois observadores e a análise volumétrica prospectiva é demonstrada pela Tabela 4 . O coeficiente de contingência para o observador 1 é $73,4 \%$, e o nível de significância é menor que $0,01 \%$. Isto implica que existe relação genuína entre as duas análises e a associação não é, portanto, resultado do acaso. Existe concordância de $83,86 \%$ entre as observações.

Tabela 4. Relação entre a análise volumétrica prospectiva e a análise visual retrospectiva dos 2 observadores.

\begin{tabular}{cccccccc}
\hline & \multicolumn{7}{c}{ Observador $1 *$} \\
\hline Análise volumétrica & D & I & E & D & I & E & Total \\
\hline D & 33 & 8 & 0 & 30 & 11 & 0 & 41,00 \\
I & 3 & 66 & 3 & 3 & 66 & 3 & 72,00 \\
E & 0 & 11 & 29 & 1 & 13 & 26 & 40,00 \\
Total & 36 & 85 & 32 & 34 & 90 & 29 & 153,00 \\
\hline
\end{tabular}

*Qui-quadrado=178,69; $<<0,0001 ; \mathrm{C}=0,734 ;$ Conc. $=83,86 \%$.

**Qui-quadrado=151,33; $\mathrm{p}<0,0001 ; \mathrm{C}=0,705 ;$ Conc. $=79,74 \%$.

$\mathrm{D}$, atrofia à direita; $\mathrm{E}$, atrofia à esquerda; I, indeterminado. 
Para o observador 2, o coeficiente de contingência é 70,5\%, e o nível de significância é menor que $0,01 \%$. Isto implica que existe uma relação genuína entre as duas análises e a associação não é, portanto, resultado do acaso. Existe concordância de $79,74 \%$ entre as observações. Verifica-se que não existe diferença significativa entre os observadores, para a análise volumétrica.

\section{DISCUSSÃO}

A neuro-imagem modificou, significativamente, o manejo de pacientes com epilepsia parcial ${ }^{26-}$ ${ }^{28}$. Graças à introdução da ressonância magnética na prática clínica, em meados da década de setenta e, mormente nos últimos anos, devido aos avanços tecnológicos, as diversas causas de ELT passaram a ser identificadas com mais facilidade do que com outros métodos de imagem, como a tomografia computadorizada $^{27,29}$. Pode-se afirmar que a RM modificou a atitude dos profissionais que lidam com epilepsias resistentes ao tratamento clínico ${ }^{26,28}$, sobretudo os portadores da síndrome epiléptica do lobo temporal. A RM é o método de escolha para a detecção das causas de desordens epilépticas, não só pela sua capacidade de demonstrar lesões expansivas ou de tecidos estranhos, mas também de sugerir, in vivo, o diagnóstico de esclerose mesial temporal, em candidatos a tratamento cirúrgico da epilepsia, dispensando, na maior parte dos casos, o emprego de métodos invasivos. Em portadores de EMT, as crises epilépticas originam-se de estruturas têmporo-mesiais e a eliminação ou melhor controle dessas crises podem ser obtidos pela ressecção cirúrgica da amígdala e da formação hipocampal ${ }^{9}$. Sabe-se que os achados de atrofia unilateral de uma formação hipocampal através da RM, seja pela análise qualitativa (visual), seja pela análise quantitativa (volumetria ou hipocampometria), correlacionam com atrofia hipocampal determinada por graus variáveis de depleção neuronal na histopatologia $a^{3,4,9}$. Além disso, atrofia hipocampal unilateral associada a perda de estrutura interna e a aumento da intensidade de sinal nas sequências ponderadas em T2 ou à redução do sinal em T1 (que correspondem à proliferação gliótica nos estudos histopatológicos) indicam uma grande possibilidade de EMT em pacientes com ELT ${ }^{4}$ (Quadro 1). Se estes achados de neuro-imagem forem congruentes com os demais (clínicos, neuropsicológicos e, sobretudo, eletrográficos), a opção cirúrgica para o tratamento da ELT pode ser proposta. O achado de EMT pela RM garante um bom prognóstico para os pacientes submetidos a lobectomia temporal ${ }^{8}$, permitindo também, em grande número de casos de epilepsia medicamente refratária, dispensar o emprego de procedimentos invasivos ${ }^{24}$.

O presente estudo avaliou, prospectivamente, o rendimento da hipocampometria ou do estudo volumétrico por RM das formações hipocampais, comparado ao de uma análise visual, baseada exclusivamente em sinais de atrofia dessas estruturas têmporo-mesiais. Uma análise visual retrospectiva das mesmas formações hipocampais foi realizada por dois observadores, de forma independente, permitindo comparação entre estes resultados e os do estudo prospectivo (análises visual e volumétrica), além de comparação entre as análises dos dois observadores. Pela análise de nossos resultados, verifica-se que a análise visual prospectiva apresenta distribuição semelhante dos casos nas classes D (atrofia direita) e E (atrofia esquerda). A classe I (indeterminada) corresponde a cerca da metade dos casos $(49,7 \%)$. Os resultados da análise volumétrica também mostram frequência semelhante entre as classes D e E. A classe I também representou a maior parte $(47,1 \%)$. A análise estatística comparativa destes resultados do estudo prospectivo demonstra que a volumetria das formações hipocampais permitiu lateralizar a atrofia em um número maior de casos, em relação à análise visual, porém esta diferença não foi estatisticamente significativa. Houve também uma boa concordância entre a análise visual retrospectiva dos dois observadores e a análise visual prospectiva. Comparando-se os resultados da análise visual retrospectiva dos dois observadores, nota-se que não houve diferença estatisticamente significativa entre ambas, embora se verifique tendência do observador $1 \mathrm{em}$ lateralizar mais casos do que o observador 2. Estes resultados mostram que a análise visual é reprodutível e constitui-se em indicador confiável de atrofia hipocampal, podendo auxiliar na lateralização da área epileptogênica em pacientes com EMT presumida clinicamente, conforme consagrado na literatura ${ }^{3,4}$. 
A análise visual pode ser suficiente e os estudos volumétricos não são imprescindíveis, pois a lateralização e o diagnóstico de EMT aumentam com a experiência do examinador. A volumetria do lobo temporal, entretanto, fornece mais informações que a análise visual das imagens por RM e permite detectar redução de volume de estruturas mesiais, que são proporcionais ao grau de alterações vistas no exame histopatológico ${ }^{9,24}$. Além do mais, relato recente refere a possibilidade de existência de esclerose hipocampal sem atrofia hipocampal detectável ${ }^{30}$. O presente estudo mostra boa concordância entre os resultados das análises visual e volumétrica, assim como entre os resultados da análise visual retrospectiva, realizada pelos dois observadores, de forma independente e utilizando os mesmos critérios de lateralização para a atrofia das formações hipocampais. Esta concordância foi significativa, tanto na comparação do estudo prospectivo (visual e volumétrico), quanto na comparação entre os resultados dos dois observadores.

Nossos resultados permitem concluir que as análises visual e volumétrica fornecem rendimento similar nos casos de atrofia hipocampal. A concordância entre as análises visual e volumétrica confirma a confiabilidade da análise visual qualitativa, como indicador da lateralização da área epileptogênica, na suspeita clínica de esclerose mesial temporal, e indica a utilidade da volumetria hipocampal como uma medida suplementar, objetiva e quantitativa, de esclerose mesial temporal. Os resultados do presente estudo reforçam a opinião consensual na literatura, de que a análise visual (qualitativa) das formações hipocampais pela RM é elemento confiável e reprodutível, quando baseada em critérios bem definidos e amplamente aceitos, sendo auxiliar valioso na lateralização da área epileptogênica em portadores de ELT. A análise objetiva, baseada em estudos volumétricos, de acordo também com critérios conhecidos, pode constituir-se em indicador adicional, sobretudo naqueles casos em que a análise visual resulte duvidosa, na suspeita de atrofia hipocampal bilateral e, sobretudo, naquele seleto grupo de pacientes com quadros epilépticos intratáveis e que venham a ser incluídos em protocolos de cirurgia de epilepsia.

O presente estudo pode indicar, ainda, algumas perspectivas de continuidade, como: comparação dos resultados da RM com outros indicadores (achados de vídeo-EEG e/ou histopatológicos, nos pacientes submetidos a cirurgia; comparação com grupo-controle, constituído de uma população não-epiléptica ou com epilepsia extratemporal; comparação com outros métodos mais recentes de neuro-imagem, com métodos qualitativos ou quantitativos; utilização de cortes de alta resolução e parâmetros 3D, visando reduzir o número de casos indeterminados. O objetivo deve ser a otimização dos critérios de lateralização da área epileptogênica em candidatos a tratamento cirúrgico da epilepsia.

Agradecimentos - À Liga das Senhoras Católicas de Curitiba, pelo suporte financeiro para a realização de vários estudos de RM desta casuística; aos Epileptologistas que encaminharam seus pacientes para o estudo.

\section{REFERÊNCIAS}

1. Bronen RA. Epilepsy: the role of MR imaging. Am J Roentgenol 1992;159:1165-1174.

2. Jackson GD. New techniques in magnetic resonance and epilepsy. Epilepsia 1994;35(Suppl 6):s2-s13.

3. Jackson GD, Berkovic SF Tress BM, et al. Hippocampal sclerosis can be reliably detected by magnetic resonance imaging. Neurology 1990;40:1869-1875.

4. Jackson GD, Berkovic SF, Duncan JS, et al. Optimizing the diagnosis of hippocampal sclerosis using MR imaging. Am J Neuroradiol 1993;14:753-762.

5. Berkovic SF, Andermann F, Olivier, A, et al. Hippocampal sclerosis in temporal lobe epilepsy demonstrated by magnetic resonance imaging. Ann Neurol 1991;29:175-182.

6. Baulac M, Saint-Hilaire JM, Adam C, et al. Correlations between magnetic resonance imaging-based hippocampal sclerosis and depht electrode investigation in epilepsy of the mesiotemporal lobe. Epilepsia 1994;35:1045-1953.

7. Falconer MA, Taylor DC. Surgical treatment of drug-resistant epilepsy due to mesial temporal sclerosis: etiology and significance. Arch Neurol 1968;19:353-361.

8. Kuzniecki R, Burgard S, Faught E, et al. Predictive value of magnetic resonance imaging in temporal lobe epilepsy surgery. Arch Neurol 1993;50: 65-69.

9. Cendes F. Ressonância magnética na avaliação da epilepsia do lobo temporal. BJECN 1995;1:7-15.

10. Baulac M, Vitte E, Dormont D, et al. The limbic system: identification of its structures on brain slices. In Gouaze A, Salamon G (eds) Brain anatomy and magnetic resonance imaging. Berlin-Heidelberg: Springer-Verlag, 1988:140-149.

11. Bronen RA. Hippocampal and limbic terminology. Am J Neuroradiol 1992;13:943-945. 
12. Naidich TP, Daniels DL, Haughton VM, et al. Hippocampal formation and related structures of the limbic lobe: anatomicMR correlation. Part 1. Surface features and coronal sections. Radiology 1987;162:747-754.

13. Mark HP, Daniels DL, Naidich TP, et al. The hippocampus. Am J Neuroradiol 1993;14:709-712.

14. Jack CR, Bentley MD, Towmey CK, et al. MR-imaging-based volume measurements of the hippocampal formation and anterior temporal lobe: validation studies. Radiology 1990;176:205-209.

15. De Carli C, Murphy DGM, Shapiro M, et al. Diagnostic utility of frontal and temporal lobe volumes as measured from magnetic resonance imaging in dementia of Alzheimer type. Neurology 1993;43:a405-a406.

16. de Leon MJ, George AE, Golomb J, et al. Hippocampal formation atrophy: a biological marker predicting Alzheimer disease. Neurology 1993;43:a405.

17. Jack CR, Towmey CK, Zinsmeister AR, et al. Anterior temporal lobes and hippocampal formations: normative volumetric measurements from MR images in young adults. Radiology 1989;172:549-554.

18. Jack CR, Sharbrough FW, Towmey CK, et al. Temporal lobe seizures: lateralization with MR volume measurements of the hippocampal formation. Radiology 1990;175:423-429.

19. Watson C, Andermann F, Gloor P, et al. Anatomic basis of amygdaloid and hippocampal volume measurements by magnetic resonance imaging. Neurology 1992;42:1743-1750.

20. Duvernoy HM. Brain anatomy. In Kuzniecki RI, Jackson GD (eds). Magnetic resonance in epilepsy. New York: Raven, 1995:49-105.

21. Dam AM. Epilepsy and neuron loss in the hippocampus. Epilepsia 1980;21:617-629.

22. Cendes F, Andermann F, Dubeau F, et al. Early childhood prolonged febrile convulsions, atrophy and sclerosis of mesial structures, and temporal lobe epilepsy: an MRI volumetric study. Neurology 1993;43:1083-1087.

23. Cendes F, Andermann F, Lopes-Cendes I et al. Atrophy of mesial structures in patients with temporal lobe epilepsy: cause or consequence of repeated seizures? Ann Neurol 1993;34:795-801.

24. Cendes F, Andermann F, Gloor P, et al. MRI volumetric measurement of amygdala and hippocampus in temporal lobe epilepsy. Neurology 1993;43:719-725.

25. Jack CR, Sharbrough FW, Cascino GD, et al. Magnetic resonance image-based hippocampal volumetry: correlation with outcome after temporal lobectomy. Ann Neurol 1992;31:138-146.

26. Gloor P. Mesial temporal sclerosis: historical background and an overview for a modern perspective. In Lüders HO (ed) Epilepsy surgery. New York: Raven Press, 1991:689-703.

27. Cascino GD, Jack CR, Parisi JE, et al. Magnetic resonance imaging-based volume studies in temporal lobe epilepsy: pathological correlations. Ann Neurol 1991;30:31-36.

28. Cascino GD. Commentary: how has the neuroimaging improved patient care ? Epilepsia 1994;35(Suppl 6):s103-s107.

29. Theodore WH, Dorwart R, Holmes M, et al. Neuroimaging in refractory partial seizures: comparison of PET, CT, and MRI. Neurology 1992;36:750-759.

30. Jackson GD, Kuzniecki RI, Cascino GD. Hippocampal sclerosis without detectable hippocampal atrophy. Neurology $1994 ; 44: 42-46$. 